\title{
Table of cases before the European Court of Justice
}

C-21-24/72, International Fruit Co NV v. Produktschap voor Groenten en Fruit, [1972] ECR $1236 \mathbf{2 0 2}$

C-127/73, BRT v. SABAM, [1974] ECR 313 47, 269, 359

C-173/73, Italy v. Commission, [1974] ECR 709 20, 42, 43, 44, 353

C-36/74, Walrave and Koch v. Association Union Cycliste International et al, (1974) ECR 1423 256, 288

C-74/76, Ianelli \& Volpi Spa v. Ditta Paola Meroni, [1977] 2 CMLR 688 36, 342

C-249/81, Commission v. Ireland, [1982] ECR 4005 256, 288

C-244/81, Commission v. Ireland, 1982, ECR 4005 36, 342

C-76/81, SA Transporoute et Travaux $v$. Minister of Public Works, [1982] ECR 457 24, 26, 45, 253, 272, 275, 345, 354, 378, 410, 423

C-323/82, Intermills v. Commission [1984] ECR 380943

C-240/83, Procureur de la République v. ADBHU, [1985] ECR 531 38, 264, 348, 359

C-152/84, Marshall v. Southampton and South West Hampshire Area Health Authority, [1986] ECR 723 257, 290
C-18/84, Commission v. France, [1985] ECR 1339 36, 342

C-103/84, Commission v. Italy, [1986] ECR 1759 36, 342

C-234/84, Belgium v. Commission, [1986] ECR 2263 40, 265, 342, 343, 349

C-24/85, Spijkers v. Gebroders Benedik Abbatoir CV, [1986] ECR 1, 1123 283, 299

C- 40/85, Belgium v. Commission. [1986] ECR I-2321 43

C-67/85, 68/85 and 70/85, Van der Kooy and Others v. Commission, [1988] ECR 219 40, 343, 350

C-199/85, Commission v. Italy, [1987] ECR 1039 398, 400, 444

C-310/85, Deufil v. Commission, [1987] ECR 901 40, 42, 43, 44, 343, 350

C-66/86, Ahmed Saeed Flugreisen v. Commission, [1989] ECR 803 269, 359, 479

C-27/86, 28/86, 29/86, Constructions et Enterprises Industrielles SA (CEI) v.

Association Intercommunale pour les Autoroutes des Ardennes, CEI and Bellini, [1987] ECR 3347 23, 272, 273, 379, 380, 396, 413

C-84/86, Commission v. Hellenic Republic, not reported 35, 341

C-27/86, Constructions et Enterprises Industrielles SA 
(CEI) v. Association

Intercommunale pour les

Autoroutes des Ardennes, C-

28/86, Ing.A. Bellini \& Co. S.p.A.

v. Regie de Betiments, C-29/86,

Ing.A. Bellini \& Co. S.p.A. v.

Belgian State, [1987] ECR 3347

23, 272, 379, 386

C-66/86, Silver Line Reisebüro, [1989] ECR I-803 269, 359, 479

C-45/87, Commission v. Ireland, [1988] ECR 4929 26, 270, 376, 377, 378, 417, 487, 496

C-31/87, Gebroeders Beentjes BV v. the Netherlands, [1989] ECR

4365 25, 30, 256, 272, 273, 279 , 282, 288, 300, 339, 380, 381, 386, 396, 408, 412, 418, 420, 484

C-45/87, Commission v. Ireland, [1988] ECR 4929 26, 270, 376, 377, 378, 417, 487, 496

C-301/87, France v. Commission, [1990] ECR I 20, 40, 343, 350

C-3/88, Commission v. Italy, [1989]

ECR 4035 372, 398, 444

C-21/88, Du Pont de Nemours

Italiana $S p A$ v. Unita Sanitaria

Locale N.2 di Carrara, [1990]

ECR 889 35, 341

C-103/88, Fratelli Costanzo S.p.A.v. Comune di Milano, [1989] ECR 1839 25, 26, 45, 275, 345, 354, 386, 410, 424

C-351/88, Lavatori Bruneau Slr. v. Unita Sanitaria Locale RM/24 di Monterotondo, [1991] ECR I3641 35, 341

C-303/88, Italy v. Commission, [1991] ECR I-1433 40, 343, 350

C-188/89, Foster v. British Gas, [1990] ECR-1313 286
C-247/89, Commission v. Portugal, [1991] ECR I-3659 286

C-261/89, Italy v. Commission, [1991] ECR I-4437 40, 343, 350

C-305/89, Italy v. Commission ('Alfa Romeo'), [1991] ECR I-1603 40, 265, 342, 343, 349

C-360/89, Commission v. Italy, [1992] ECR I-3401 30, 35, 279, 341

C-296/89, Impresa Dona Alfonso di Dona Alfonso \& Figli s.n.c. v. Consorzio per lo Sviluppo Industriale del Comune di Monfalcone, judgment of 18 June 1991 25, 26, 45, 275, 345, 353, 354, 410, 424

C-288/89, Stichting Collectieve Antennevoorziening Gouda and others v. Commissariaat voor de Media, [1991] ECR I-04007 478

C-41/90, Höfner, [1991] ECR I-1979 479

C-179/90, Merci Convenzionali Porto di Gevova, [1991] ECR 15889 15, 261, 293

C-362/90, Commission v. Italy, judgment of 31 March $1992 \mathbf{3 5 , 3 4 1}$

C-24/91, Commission v. Kingdom of Spain, [1994] CMLR 621 286, 398, 407, 444

C-29/91, Dr Sophie Redmond Stichting v. Bartol, IRLR 369 283, 299

C-209/91, Rask v. ISS Kantinservice, [1993] ECR 1 283, 299

C-107/92, Commission v. Italy, judgment of 2 August 1993 398, 407, 444

C-296/92, Commission v. Italy, judgment of 12 January $1994 \mathbf{3 9 8 ,}$ 444 
C-71/92, Commission v. Spain, judgment of 30 June $1993 \mathbf{2 7 3 ,} \mathbf{3 8 0 ,}$ 396, 403

C-89/92, Ballast Nedam Groep NV v. Belgische Staat, [1994] 2 CMLR 379

C-278/92 to C-280/92, Spain v. Commission, [1994] ECR I-4103 40, 343, 349

C-296/92, Commission v. Italy, judgment of 12 January $1994 \mathbf{3 9 8 ,}$ 444

C-364/92, SAT Fluggesellschafeten, [1994] ECR 1-43 262, 294

C-382/92, Commission v. United Kingdom, [1994] ECR 1 283, 299

C-387/92, Banco Exterior, [1994]

ECR I-877 38, 40, 264, 343, 348

C-392/92, Schmidt v. Spar und Leihkasse der fruherer Amter Bordersholm, Kiel und Cronshagen, [1994] ECR 1, 1320 283, 299

C-382/92, Commission v. United Kingdom, [1994] ECR 1 283, 299

C-56/93, Belgium v. Commission, [1996] ECR I-723 40, 42, 43, 343, 350, 353

C-359/93, Commission v. the Netherlands, judgment of 24 January 1995 26, 200, 270, 376, 378

C-280/93, Germany v. Council, judgment of 5 October 1994 202, 388

C-324/93, R. v. The Secretary of State for the Home Department, ex parte Evans Medical Ltd and Macfarlan Smith Ltd, judgment of 28 March 1995 273, 282, 412

C-392/93, The Queen and H.M. Treasury, ex parte British
Telecommunications PLC, OJ 1993, C 287/6 46, 263, 287, 357

C-39/94, SFEI and Others, [1996]

ECR I-3547 40, 343, 350

C-48/94, Rygaard v. Stro Molle Akustik, judgment of 19

September 1995 283, 299

C-57/94, Commission v. Italy, judgment of 18 May 1995 398, 400, 403, 444

T-67/94, Ladbroke Racing $v$. Commission, [1998] ECR II-1 40, 343, 350

C-79/94, Commission v. Greece, judgment of 4 May 1995200

C-87/94R, Commission v. Belgium, order of 22 April 1994 372, 384, 409, 497

C-157/94, Commission v Netherlands, [1997] ECR I-5699. 47, 269, 359

C-158/94, Commission v. Italy, [1997] ECR I-5789 47, 269, 359

C-159/94, Commission v. France, [1997] ECR I-5815 47, 269, 359

C-160/94, Commission v. Spain, [1997] ECR I-5851 47, 269, 359

C-241/94, France v. Commission, [1996] ECR I-4551 42, 43, 44, 353

T-358/94, Air France v. Commission, [1996] ECR II-2109 40, 343, 350

T-106/95, FFSA and Others $v$. Commission, [1997] ECR II-229

38, 264, 348

C-343/95, Diego Cali et Figli, [1997] ECR 1-1547 262, 294, 484

T-16/96, Cityflyer Express $v$. Commission, [1998] ECR II- 757 40, 43, 343, 350 
C-44/96, Mannesmann Anlangenbau Austria AG et al. v. Strohal Rotationsdurck GesmbH, [1998] ECR 73 11, 18, 29, 42, 258, 261, 266, 278, 293, 300, 301, 307, 309, 312, 317, 322, 323, 324, 339, 345, 347, 352, 412, 482, 284

C-323/96, Commission v. Kingdom of Belgium, [1998] ECR I-5063 256, 289, 399

C-342/96, Spain v. Commission, [1999] ECR I-2459 40, 343, 350

C-353/96, Commission v. Ireland and C-306/97, Connemara Machine Turf Co Ltd v. Coillte Teoranta, [1998] ECR I-8565 256, 288

C-360/96, Gemeente Arnhem Gemeente Rheden v. BFI Holding $B V$, [1998] ECR $6821 \mathbf{1 1 , 1 7 , 2 9 ,}$ 41, 261, 262, 266, 278, 293, 294, 300, 305, 307, 312, 315, 324, 334, 335, 337, 339, 343, 346, 350, 412, 435, 447, 448, 450, 482, 484

C-5/97, Ballast Nedam Groep NV v. Belgische Staat, judgment of 18 December 1997379

C-6/97, Italy v. Commission, [1999] ECR I-2981 40, 343, 350

T-46/97, [2000] ECR II-2125 38, 264, 348

C-75/97, Belgium v. Commission, [1999] ECR I-3671 40, 343, 350

C-144/97, Commission v France, [1998] ECR 1-613 289

C-174/97 P, [1998] ECR I-1303 38, 364, 348

T-204/97 and T-270/97, EPAC v.

Commission, [2000] ECR II-2267 40, 343, 350
C-256/97, DM Transport, [1999] ECR I-3913 40, 343, 350

T-613/97, Ufex and Others $v$. Commission, [2000] ECR II-4055 41, 266, 351

T-266/97, Vlaamse Televisie Maatschappij, [1999] ECR II2329479

C-124/97, Markku Juhani Läärä, Cotswold Microsystems Ltd and Oy Transatlantic Software Ltd v Kihlakunnansyyttäjä (Jyväskylä) and Suomen valtio (Finnish State), [1999] ECR I-06067 478

C-107/98, Teckal Slr. v. Commune di Viano, [1999] ECR I-8121 46, 257, 260, 268, 292, 298, 303, $325,339,356,364,385,392$, 467, 484

C-156/98, Germany v. Commission, [2000] ECR I-6857 40, 343, 350

C-176/98, Holst Italia v. Comune di Cagliari, [1999] ECR I-8607

340, 341, 379, 389

C-380/98, The Queen and H.M. Treasury, ex parte University of Cambridge, [2000] ECR 8035 29, 217, 257, 278, 290, 292, 314, 320, 325, 371, 412, 484

C-324/98, Telaustria Verlags GmbH, Telefonadress $\mathrm{GmbH}$ and Telekom Austria AG, [2000] ECR I-10745 217, 334, 336, 338, 369, 371, $372,373,383,395,408,435$, 446, 449, 450, 484, 497

C-94/99, ARGE Gewässerschutzt v. Bundesministerium für Land- und Forstwirtschaft, [2000] ECR I11037 32, 45, 276, 302, 345, 354, 380, 411, 424

C-223/99, Agora Srl v. Ente Autonomo Fiera Internazionale di 
Milano and C-260/99 Excelsior Snc di Pedrotti Runa \& C v. Ente Autonomo Fiera Internazionale di Milano, [2001] ECR 3605 11, $18,41,261,262,266,293,294$, $325,339,343,350,482,484$

C-237/99, Commission v. France, [2001] ECR 934 29, 257, 278, 290, 292, 312, 315, 325, 412, 484

C-285/99 \& 286/99, Impresa

Lombardini SpA v. ANAS, [2001] ECR I-9233 25, 26, 276, 383, 393, 408, 424, 484

C-513/99, Concordia Bus Finlandia v. Helsingin Kaupunki et HKLBussiliikenne, [2000] ECR I-7213 25, 274, 283, 381, 384, 406, 414, 415, 484

C-470/99, Universale-Bau AG, Bietergemeinschaft, ECR [2002] I-11617 205, 224, 228, 239, 246, $285,301,312,313,320,322$, 323, 407, 408, 421, 484

C-53/00, Ferring SA v. Agence centrale des organismes de s? curit? sociale (ACOSS), [2001] ECR I-09067 38, 264, 348

C-280/00, Altmark Trans GmbH and Regierungspräsidium Magdeburg v. Nahverkehrsgesellschaft Altmark GmbH and Oberbundesanwalt beim Bundesverwaltungsgericht, [2003] ECR 1432 38, 39, 264, 268, 348, 358

C-214/00, Commission of the European Communities v. Kingdom of Spain, ECR [2003] I4667 207, 241, 301, 309, 322, 323

C-92/00, Hospital Ingenieure Krankenhaustechnik Planungs-
$\mathrm{GmbH}(\mathrm{HI})$ and Stadt Wien, ECR [2002] I-5553 213, 214, 227, 229, 233, 243, 245, 285, 371, $395,408,487,496$

C-283/00, Commission of the European Communities $v$. Kingdom of Spain, [2003] ECR I11697 301, 303, 322, 324

C-327/00, Santex SpA and Unità Socio Sanitaria Locale n. 42 di Pavia, [2003] ECR I-1877 232, 244, 246

C-373/00, Adolf Truley GmbH and Bestattung Wien GmbH, [2003] ECR I-1931 292, 310, 312, 323, 346, 484

C-411/00, Felix Swoboda GmbH and Österreichische Nationalbank, [2002] ECR I-10567 360, 362

C-5/01, Belgium v. Commission, [2002] ECR I-3452 42, 43, 44, 231, 240, 353, 380, 382, 481, 484

C-83/01 P, C-93/01 P and C-94/01. Chronopost and Others, [2003] ECR I-6993 41, 266, 351

C-126/01, Ministre de l'economie, des finances et de l'industrie $v$. GEMO SA, [2003] ECR $3454 \mathbf{4 2}$

C-314/01, Siemens AG Österreich, ARGE Telekom \& Partner and Hauptverband der österreichischen Sozialversicherungsträger, [2004] ECR I-2549 219, 340, 341, 389, 484

C-448/01, EVN AG, Wienstrom GmbH and Republik Österreich, [2003] ECR I-14527 220, 274, 419, 422

C-249/01, Werner Hackermüller and Bundesimmobiliengesellschaft mbH (BIG), Wiener Entwicklungsgesellschaft $\mathrm{mbH}$ für 
den Donauraum AG (WED), ECR [2003] I-6319 223, 226, 242

C-315/01, Gesellschaft für Abfallentsorgungs-Technik GmbH (GAT) and Österreichische Autobahnen und Schnellstraßen $A G$ (ÖSAG), ECR [2003] I-6351 231, 240, 380, 382, 421, 484

C-410/01, Fritsch, Chiari \& Partner, Ziviltechniker GmbH and Others and Autobahnen- und Schnellstraßen-Finanzierungs-AG (Asfinag), ECR [2003] I-11547 204, 205, 224, 225, 244

C-421/01, Traunfellner GmbH and Österreichische Autobahnen- und Schnellstraßen-Finanzierungs-AG (Asfinag), [2003] ECR I-11941 418, 419

C-18/01, Arkkitehtuuritoimisto Riitta Korhonen Oy, Arkkitehtitoimisto Pentti Toivanen Oy,

Rakennuttajatoimisto Vilho Tervomaa and Varkauden Taitotalo Oy, [2003] ECR I-5321 310, 316, 323, 324, 340, 484 Joined Cases C-20/01 and C-28/01, Commission of the European Communities v. Federal Republic of Germany, [2003] ECR I-3609 248, 404, 407

C-57/01, Makedoniko Metro, Mikhaniki AE and Elliniko Dimosio, [2003] ECR 1-1091 213, 227, 233, 390, 394, 484, 487, 496,

C-243/01, Gambelli, [2003] ECR I 13031478

C-230/02, Grossmann Air Service, Bedarfsluftfahrtunternehmen GmbH \& Co. KG and Republik Österreich, [2004] ECR I-1829 204, 223, 244, 245
C-212/02, Commission of the European Communities $v$. Republic of Austria, unpublished 206, 222, 242, 244, 285

Joined Cases C-21/03 and C-34/03, Fabricom SA v. État Belge, not yet reported.

C-84/03, Commission of the European Communities v. Kingdom of Spain, not yet reported 383, 384, 385, 388, 484, 487, 496

C-26/03, Stadt Halle, RPL Recyclingpark Lochau GmbH v. Arbeitsgemeinschaft Thermische Restabfall- und Energieverwertungsanlage TREA Leuna, not yet reported 212, 214, 222, 243, 244, 301, 323, 327, 329, 331, 339, 340, 389, 465, 468, 484

C-126/03, Commission of the European Communities v. Federal Republic of Germany, [2005] ECR I-00001 339, 340, 389, 484

C-264/03, Commission v. France, [2005] ECR I-8831 366, 476, 487, 496

C-458/03, Parking Brixen GmbH v. Gemeinde Brixen and Stadtwerke Brixen AG, [2005] ECR I-08585 326, 327, 329, 369, 372, 469, 497

C-84/03, Commission v. Spain, [2005] ECR-I00139 300, 363, 402, 484

C-231/03, Consorzio Aziende

Metano (Coname) v. Comune di Cingia de' Botti, [2005] ECR I07287 329, 369, 370, 371, 372, 470, 496

C-264/03, Commission v. France, 
[2005] ECR I-08831 366, 369, 476, 487, 496

C412/04, Commission v. Italy, [2008] ECR I0000 369

C-29/04, Commission v. Austria, [2005] ECR I-09705. 245, 328, 329, 468

C-340/04, Carbotermo SpA and Consorzio Alisei v. Comune di Busto Arsizio and AGESP SpA, [2006] ECR I-04137 326, 327, 330,469

C382/05, Commission v. Italy, [2007] ECR I6657 373

C-6/05, Medipac-Kazantzidis AE v. Venizelio-Pananio (PE.S.Y. KRITIS), [2007] ECR I4557 369

C-295/05, Asociación Nacional de Empresas Forestales (Asemfo) v. Transformación Agraria SA (Tragsa) and Administración del Estado, [2007] ECR I-2999 327, 331, 477

C-371/05, Commission v. Italy, [2008] ECR I-00110 468

C-532/06, Emm G. Lianakis AE v. Alexandroupolis, [2008] ECR I251396

C-454/06, Pressetext

Nachrichtenagentur, [2008] ECR I4401 374

C-241/06, Lämmerzahl GmbH v. Freie Hansestadt Bremen, [2008] 1 CMLR 462246

C-70/06, Commission v. Portugal, [2004] ECR I-0000 249

C-492/06, Consorzio Elisoccorso

San Raffaele v. Elilombarda Srl and Azienda Ospedaliera Ospedale Niguarda Ca' Granda di Milano, [2007] ECR I-08189 245
C-220/06, Asociación Profesional de Empresas de Reparto y Manipulado de Correspondencia $v$ Administración General del Estado, [2007] ECR I-12175 369, 477, 479

C147/06 and C-148/06, SECAP SpA and Santorso Soc. coop. arl, [2008] ECR I-3565 369

C-480/06, Commission v. Germany, [2009] ECR I-04747 331, 367

C437/07, Commission v. Italy, [2008] ECR I0000 373, 463

C-423/07, Commission v. Spain, judgment of 22 April $2010 \mathbf{3 7 5}$

C-536/07, Commission v. Germany, ECR-I 10355365

C-324/07, Coditel Brabant SA v. Commune d'Uccle and Région de Bruxelles- Capitale, [2008] ECR I-08457 327, 332, 467

C-573/07, Sea Srl v. Comune di Ponte Nossa, [2009] ECR I08127 328, 467

C-42/07, Liga Portuguesa de Futebol Profissional and Bwin International Ltd $v$.

Departamento de Jogos da Santa Casa da Misericórdia de Lisboa, [2009] ECR I-07633 478

C-206/08, WAZV Gotha v.

Eurawasser Aufbereitungs, [2009] ECR I-8377 373, 463, 464

C-160/08, Commission v. Germany, judgment of 29 April 2010374

C-91/08, Wall AG v. Stadt Frankfurt am Main, judgment of 13 April 2010 248, 374

C-145/08 and C-149/08, Club Hotel Loutraki AE and Others v. Ethnico Symvoulio Radiotileorasis [2010] All ER (D) 249 
C-149/08, Aktor Anonymi Techniki Etaireia (Aktor ATE) v. Ethnico Symvoulio Radiotileorasis, [2010] ECR I-0000 249

C-406/08, Uniplex (UK) Ltd v. NHS Business Services Ltd, [2010] ECR I-0000 247

C-456/08, Commission v. Ireland, [2010] ECR I-0000 247 C-451/08, Helmut Müller v. Bundesanstalt für
Immobilienaufgaben, [2010] 3 CMLR $18 \mathbf{3 6 8}$

C-5/08, Commission v. Germany, [2009] ECR I-00168 249

C-03/08, The Sporting Exchange Ltd, trading as Betfair v. Minister van Justitie, [2010] not yet published 462, 478

C-17/09, Commission v. Germany, judgment of 21 January 2010 247 